\title{
The multiligand receptor RAGE as a progression factor amplifying immune and inflammatory responses
}

\author{
Ann Marie Schmidt, ${ }^{1,2}$ Shi Du Yan, ${ }^{3}$ Shi Fang Yan, ${ }^{1}$ and David M. Stern ${ }^{1,4}$ \\ ${ }^{1}$ Department of Surgery, \\ ${ }^{2}$ Department of Medicine, \\ ${ }^{3}$ Department of Pathology, and \\ ${ }^{4}$ Department of Physiology and Cellular Biophysics, College of Physicians and Surgeons of Columbia University, New York, New York, USA \\ Address correspondence to: David Stern, Department of Surgery, P\&S 17-401, College of Physicians and Surgeons of Columbia University, \\ 630 West 168th Street, New York, New York 10032, USA. Phone: (212) 305-1615; Fax: (212) 305-5337; E-mail: dms9@columbia.edu.
}

J. Clin. Invest. 108:949-955 (2001). DOI:10.1172/JCI200114002.

The multiligand receptors that form the focus of this Perspective series have expectedly diverse functions, often conforming to potential gaps in the host response to invading pathogens that are not effectively manned by adaptive immunity. For example, the macrophage scavenger receptor (type A) interacts with bacterial cell walls and enhances clearance of Gramnegative bacteria from the circulation (1). Similarly, the macrophage mannose receptor binds mannose-rich carbohydrates typical of many microorganisms, thereby promoting their cellular uptake and disposal (2). The present contribution to the series concerns a member of the immunoglobulin superfamily that differs from the above molecules in that all known ligands in its broad repertoire can be generated endogenously (3). This cell surface protein, called RAGE because it serves as a receptor for nonenzymatically glycated adducts termed "advanced glycation endproducts" (AGEs), also binds $\beta$-sheet fibrils characteristic of amyloid; proinflammatory cytokine-like mediators of the S100/calgranulin family; and amphoterin, a nuclear protein sometimes found in the ECM (Table 1).

Binding of these ligands to RAGE does not accelerate clearance or degradation but rather begins a sustained period of cellular activation mediated by receptordependent signaling. This is the first of several distinctive themes that have emerged from studies of RAGE. Other unusual features of the receptor include its ability to engage classes of molecules, rather than individual ligands, and its enhanced surface expression in environments rich in RAGE ligands. This last point is crucial, since it explains how upregulation of this receptor can contribute to an ascending spiral of RAGEdependent cellular perturbation. Taken together, these features of RAGE allow the receptor to propagate cellular dysfunction in a number of pathophysiologically relevant situations, most often dictated by the formation and persistence of ligands in the tissues. As described below, these diverse situations range from the complications of diabetes and cellular perturbation in amyloidoses to immune and inflammatory responses and tumor cell behavior.

RAGE and the complications of diabetes

Nonenzymatic glycoxidation of macromolecules ultimately resulting in the formation of AGEs is enhanced at sites of inflammation, in renal failure, in the presence of hyperglycemia, and under other conditions associated with systemic or local oxidant stress (4). Accumulation of AGEs in the vasculature can occur focally, as in the joint amyloid composed of AGE- $\beta 2$-microglobulin found in patients with dialysis-related amyloidosis (5), or generally, as exemplified by the vasculature and tissues of patients with diabetes (6). The progressive accumulation of AGEs in patients with diabetes suggests that endogenous clearance mechanisms fail to function effectively at sites of AGE deposition. Such deposited AGEs have the capacity to alter cellular properties by a number of mechanisms. Direct effects of AGEs in the extracellular space include formation of crosslinks that can also trap neighboring unrelated macromolecules (4). Nonenzymatic glycation has been shown to alter properties of FGF, as well as vitronectin and laminin (4). However, AGE engagement of cellular receptors would provide a biologic amplification mechanism whereby glycoxidized adducts could modulate properties of multiple cells.

RAGE was first identified from tissue homogenates based on its capacity to bind a glycoxidized form of

Table 1

RAGE ligands and associated pathophysiologic states

Diabetic complications

AGEs

S100/calgranulins

Amyloidoses

$\beta$-sheet fibrils

S100/calgranulins AGEs

Immune/inflammatory disorders

S100/calgranulins Amphoterin AGEs

Tumor biology

Amphoterin 


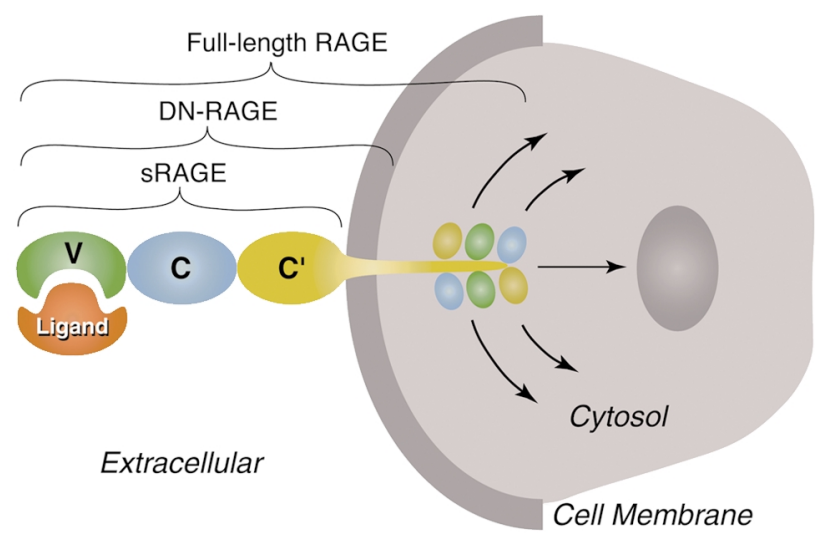

Figure 1

Schematic depiction of RAGE-ligand interaction showing domains of the receptor. The V-type domain is critical for binding of RAGE ligands. Deletion of the cytosolic tail results in a modified form of RAGE that binds ligands, remains firmly embedded in the cell membrane, but does not transmit RAGE-mediated cellular activation. Even in the presence of endogenous full-length RAGE, expression of dominant negative RAGE blocks signaling from the receptor. SRAGE, soluble RAGE (extracellular domain of RAGE); DN-RAGE, dominant negative RAGE (extracellular domain + transmembrane-spanning domain)

albumin prepared in vitro (7). Its extracellular domain consists of three immunoglobulin-like regions, one "V"-type followed by two "C"-type (8). RAGE contains a single transmembrane-spanning domain and a 43-amino acid cytosolic tail. Structure-function studies have shown that the $\mathrm{V}$-domain is critical for ligand binding and that the cytosolic tail is essential for RAGE-mediated intracellular signalling (Figure 1). A truncated form of RAGE, which lacks the cytosolic tail, remains firmly embedded in the membrane. Although this form of the receptor is competent to bind the usual complement of RAGE ligands, it acts as a dominant negative (termed "DN-RAGE") receptor, and its expression strikingly suppresses RAGE-mediated signaling, even in cells bearing the full-length form $(9,10)$.

RAGE is expressed at low levels in normal tissues and vasculature. However, the receptor becomes upregulated wherever its ligands accumulate (11-13). In diabetic vessels, for example, RAGE ligands include AGEs of at least two types, (carboxymethyl)lysine adducts and hydroimidazolones (14), and S100/calgranulins as well. RAGE expression is increased in endothelium, smooth muscle cells, and infiltrating mononuclear phagocytes in diabetic vasculature. The overlapping expression of RAGE and its ligands led us to consider the possibility that RAGE mediates cellular activation in diabetic vessels. Indeed, studies in cell culture show that AGE-RAGE interaction alters cellular properties important in vascular homeostasis (15-17). For example, following engagement of RAGE by AGEs, endothelia increase their expression of VCAM-1, tissue factor, and IL-6, and their permeability to macromolecules (18-20). AGE-RAGE-mediated activation of the transcription factor NF- $\kappa \mathrm{B}$ is likely to explain these observations, at least in part $(20,21)$. In mononuclear phagocytes, RAGE activates expression of cytokines and growth factors and induces cell migration in response to soluble AGEs, whereas haptotaxis occurs with immobilized ligands (22).

These considerations led us to propose the following two-hit model for RAGE-mediated perturbation of cellular properties in diabetic vasculature: the presence of ligands for RAGE changes properties of the vasculature, resulting in a basal state of activation/dysfunction (first hit; Figure 2); with a superimposed stimulus, such as deposition of oxidized lipoproteins, infection, or ischemia, cellular responses favor derangement of vascular function and tissue damage, rather than restitution of homeostasis (second hit; Figure 2).

Diabetic macrovascular disease provides an especially important situation to test this model, as epidemiologic studies have suggested that factors other than glycemia and those traditionally associated with atherosclerosis contribute to pathogenesis. For these studies, our initial experimental system employed atherosclerosis-prone mice (apoE-null animals in the C57BL/6 background) treated with streptozotocin (23), a $\beta$-cell toxin that causes insulin deficiency. In contrast to euglycemic apoE-null mice, diabetic animals display advanced atherosclerosis already evident by 14 weeks of age. Diabetic vasculature in the apoEnull animals shows an increased number and area of atherosclerotic lesions, as well as enhanced complexity, with fibrous caps, cholesterol clefts, and necrosis. Levels of AGEs and RAGE are increased in diabetic vasculature, as is expression of tissue factor, VCAM-1, and matrix metalloproteinases (MMPs) 2 and 9. To deter-
Hit 1

RAGE-Ligand Interaction
Hit 2

Ischemic Stress Immune/Inflammatory Stimuli Modified Lipoproteins Physical Stress

\section{Figure 2}

Schematic depiction of a two-hit model of vascular perturbation mediated by RAGE and its ligands. We hypothesize that the diabetic vessel wall displays increased expression of RAGE ligands and the receptor itself (first hit). In the presence of additional perturbation (second hit), such as ischemic stress, immune/inflammatory stimuli, physical stress, or modified lipoproteins, there is an exaggerated cellular response promoting formation of vascular lesions (rather than restitution of vascular homeostasis).

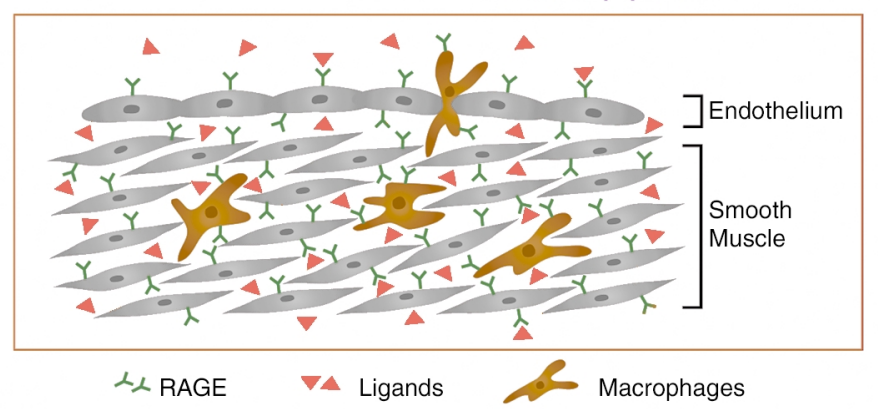


mine whether blocking access of ligands to cell surface RAGE affects the accelerated atherosclerosis seen in the diabetic animals, we used a soluble, truncated form of RAGE (sRAGE), spanning the receptor's ligand-binding extracellular domain (Figure 1) (19). As low levels of sRAGE are present in the plasma normally, we could administer recombinant sRAGE to animals daily to achieve microgram levels in the plasma without triggering an immune response. We found that treatment of diabetic apoE null mice with sRAGE suppressed the accelerated pace of atherogenesis down to the level observed in euglycemic controls in a dose-dependent manner (23). Furthermore, lesion complexity in sRAGE-treated diabetic mice was reduced, and the remaining lesions were mostly fatty streaks, resembling the level of complexity observed in euglycemic apoEnull mice at the same early age.

Vessels from animals treated with sRAGE display reduced levels of cellular RAGE and AGEs. AGEs can be detected in the plasma complexed with sRAGE, but such complexes do not appear to accumulate and are presumed to be degraded. Inhibition of AGE-RAGE interaction might also affect formation of AGEs, since RAGE-mediated cellular activation promotes glycoxidation and other manifestations of oxidant stress. As the level of RAGE ligands in the vessel wall declines, so does expression of RAGE and of vascular activation markers associated with atherosclerosis. It is important to note that the reduced pace of atherogenesis in diabetic mice treated with sRAGE does not involve changes in glycemia or plasma cholesterol or triglycerides.

To determine whether our findings could be extrapolated to other experimental models, and to establish a model system relevant to insulin resistance, we have begun working with genetically diabetic $(d b / d b)$ mice. Our preliminary studies have shown that $d b / d b$ animals deficient in apoE display accelerated atherosclerosis which can be suppressed by administration of sRAGE (24), thus extending our results from the streptozotocin-induced diabetes model (above). Blockade of RAGE using sRAGE may therefore be broadly applicable for treating atherogenesis in diabetic vasculature.

Amplification of the inflammatory response in murine models of diabetic atherosclerosis, as a consequence of the RAGE-ligand interaction, has also proven applicable to the pathogenesis of tissue injury in other settings of diabetic complications. Diabetic wounds are well known to be slow to close, poor at forming granulation tissue, and prone to sustained inflammation. We have found that administration of sRAGE in a murine diabetic wounding model leads to accelerated wound closure most likely by decreasing expression of proinflammatory cytokines and matrix metalloproteinases, and by augmenting levels of angiogenic factors (25). In diabetic periodontal disease, impaired host response mechanisms in the diabetic subject must contend with invading Gram-negative pathogens as well as destruction of periodontal tissues by host effector pathways. Blockade of RAGE in a murine model of diabetic periodontal disease diminishes the loss of alveolar bone while blocking the destructive inflammatory process (26). Containment of the Gram-negative infection also occurred effectively in the presence of sRAGE. These results suggest that RAGE-ligand interaction provides a chronic inflammatory pathway contributing to the pathogenesis of diabetic complications. The consequences of triggering such pathways include the recruitment of inflammatory mediators and effector molecules (for example, sustained expression of TNF- $\alpha$, IL-1, IL-6, and metalloproteinases) eventuating in accelerated tissue destruction. These considerations suggest the possibility that blockade of RAGE could provide an effective approach in a range of diabetic complications.

RAGE and cellular dysfunction in the amyloidoses Deposition of amyloid in tissues displaces normal structures and, at high concentrations, can exert nonspecific toxic effects on cells by disturbing the integrity of membranes. The latter, traditional view of amyloid is being replaced by the concept that amyloid deposits and low-molecular weight amyloid fragments are biologically active via their interaction with specific cell surface receptors (27-31). Such receptors appear to act early in the disease process when the amyloid burden is low, possibly by amplifying the response to nascent amyloid (Figure 3). RAGE binds $\beta$-sheet fibrillar material regardless of the composition of the subunits (amyloid- $\beta$ peptide, $A \beta$, amylin, serum amyloid $A$, and prion-derived peptides, among others) $(27,28)$, and deposition of amyloid results in enhanced expression of RAGE. For example, in the brains of patients with Alzheimer disease (AD), RAGE expression increases in neurons and glia. The consequences of $A \beta$ ligation of RAGE appear to be quite different on neurons versus microglia. Whereas microglia become activated as a consequence of $A \beta$-RAGE interaction, as reflected by increased motility and expression of cytokines (27), early RAGE-mediated neuronal activation is superseded by cytotoxicity at later times (33). Thus, in our preliminary results, transgenic mice with targeted neu-

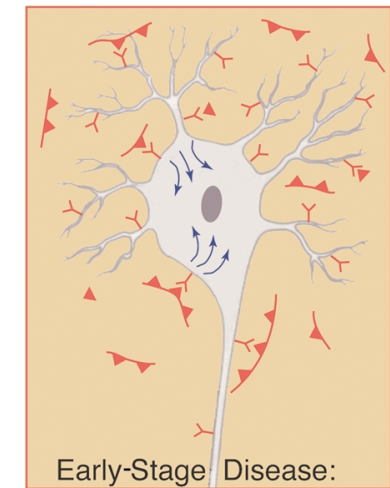

Receptor-Dependent

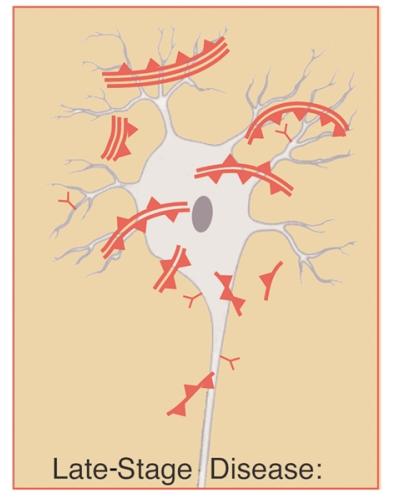

Receptor-Independent
Receptor for $A \beta$

\section{Figure 3}

Schematic depiction of a role for receptor-mediated interaction with pathogenic $A \beta$ assemblies (which could be dimers, multimers, and/or fibrils) in mediating cellular dysfunction early in amyloidoses (left). In contrast, nonspecific neuronal/cellular toxicity (for example, interfering with the integrity of cell membranes) is likely to be an important mechanism of cellular injury at later stages of the disease when there is a higher level of $A \beta$ (right). 


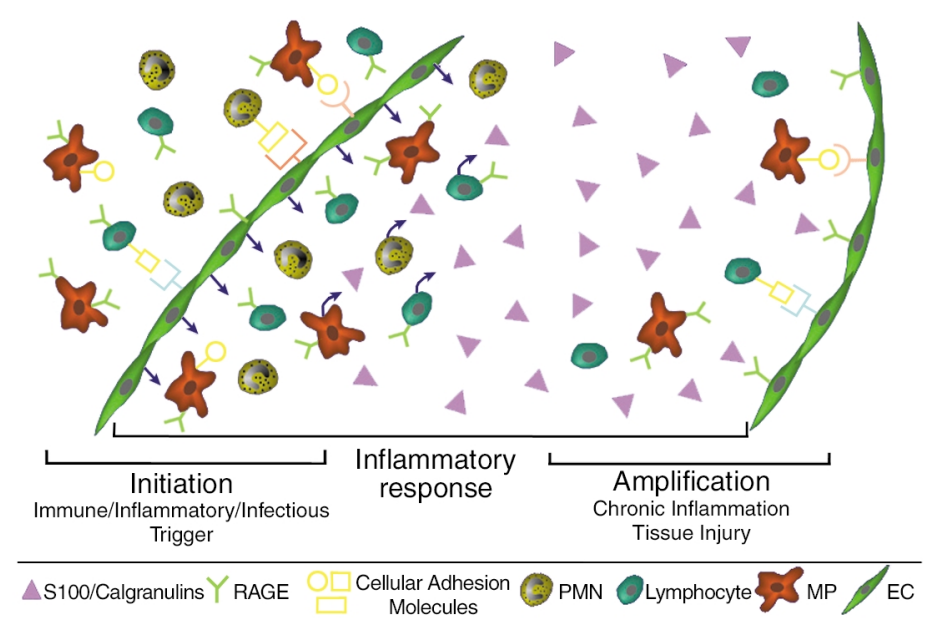

Figure 4

Schematic depiction of a role for RAGE and its ligands (especially S100/calgranulins) at the site of a chronic immune/inflammatory response. We hypothesize that S100/calgranulin-RAGE interaction provides a mechanism amplifying the inflammatory response by mediating activation of RAGE-bearing cells, including mononuclear phagocytes, lymphocytes, and cells in the vessel wall. In contrast, RAGE and its ligands are not the trigger for initiating the host response, which may be due to primary immune, inflammatory, or infectious stimuli.

with AGEs and amyloid. We sought other endogenous ligands present in tissues that might engage the receptor, as a part of protective host response mechanisms. As RAGE is most abundant in the lung, we screened lung extracts for polypeptides that bind specifically to RAGE.

ronal expression of RAGE and mutant amyloid precursor protein - the latter causing an $\mathrm{A} \beta$-rich environment in the brain - display exaggerated cell stress at 3-5 months, including activation of NF- $\mathrm{KB}$ and expression of IL-6. At 8-10 months, cytotoxicity appears to be increased (34). These preliminary findings are consistent with RAGE-mediated amplification of the effects of $A \beta$ even prior to extensive amyloid deposition. Inhibition of $A \beta$-induced cerebral vasoconstriction and reduced transfer of the amyloid peptide across the blood-brain barrier following receptor blockade provide further evidence of a role for RAGE in cellular interactions with $\mathrm{A} \beta$ (35).

To analyze RAGE-amyloid interactions more directly, we have turned to a model of systemic amyloidosis in which the effects of RAGE blockade can be assessed (28). In the spleen, the distribution of amyloid A overlaps closely with that of RAGE, which is especially prominent in mononuclear phagocytes. Nuclear extracts of splenic tissue showed NF- $\mathrm{\kappa B}$ activation and increased expression of heme oxygenase type 1, M-CSF, and IL-6 (28). In contrast to A $\beta$ amyloid, which is sequestered behind the blood-brain barrier in $\mathrm{AD}$, amyloid A and increased levels of cellular RAGE in the spleen are accessible to sRAGE or anti-RAGE F( $\left.\mathrm{ab}^{\prime}\right)_{2}$ molecules. Following receptor blockade with these agents, we observed decreased NF- $\mathrm{KB}$ activation and reduced expression of cellular RAGE and cell stress markers, as well as a loss of amyloid (28), suggesting that interactions with RAGE induce or maintain both the deposition of amyloid and the altered cellular properties seen in amyloid-rich environments.

Taken together, these data lead us to propose that in amyloidoses the interaction of RAGE with pathologic $A \beta$ species (as well as other amyloids) provides an early event modulating properties of the vasculature and neurons. This interaction also apparently primes host response mechanisms through its effects on cells of mononuclear phagocyte lineage.

\section{RAGE and propagation}

of the immune/inflammatory response

Based on the emerging multiligand nature of RAGE, it seemed unlikely that the receptor would solely interact
These studies resulted in the identification of S100/calgranulins and amphoterin as ligands of RAGE (36).

The S100/calgranulins constitute a family of closely related calcium-binding polypeptides containing two EF-hand regions, linked by a connecting peptide (37). Although they lack signal peptides, it has long been known that S100/calgranulins gain access to the extracellular space, especially at sites of chronic immune/inflammatory responses, as in cystic fibrosis and rheumatoid arthritis. Because the S100/calgranulins appear to act as proinflammatory cytokines (37), it was expected that specific cell surface receptors must mediate their effects, and we have shown that RAGE binds S100A12 (also termed EN-RAGE for extracellular, newly discovered RAGE ligand) and the related protein S100B, with nanomolar affinity (9). These findings lead us to predict that RAGE is a receptor for many members of the S100/calgranulin family, mediating their proinflammatory effects on cells such as lymphocytes and mononuclear phagocytes. Indeed, addition of S100A12 to lymphocytes results in expression of IL-2 and cell proliferation; mononuclear phagocytes elaborate IL- $1 \beta$ and TNF- $\alpha$ under the same conditions (9). An emerging view of RAGE in immune/inflammatory responses is shown in Figure 4. We propose that RAGE-S100 interaction provides a novel costimulatory axis in which aggregates of $\mathrm{S} 100$ proteins bridge and activate RAGE on lymphocytes and monocytes. Experiments are underway to test this hypothesis.

The delayed-type hypersensitivity response provided a logical model in which to test the effect of RAGES100 interaction on cellular activation in the context of immunity and inflammation. Animals sensitized and challenged with methylated BSA, which is not a ligand of RAGE, show marked lower-extremity swelling and infiltration of the tissue with inflammatory cells, as well as activation of NF- $\mathrm{KB}$ and expression of proinflammatory cytokines. Levels of S100/calgranulins and RAGE in these inflammatory lesions are also strikingly increased. Administration of sRAGE to animals at the time of the challenge dose of methylated albumin strikingly decreases infiltration of the tissue by immune/inflammatory effector cells, as well as other associated changes (edema, expression of cytokines, 
etc.) (9). Blocking antibodies to $S 100$ proteins or to RAGE itself also suppress pathologic changes, and the most prominent suppression is observed following treatment with both antibodies together, presumably because the dual treatment allows a more complete blockade of RAGE-ligand interaction.

These results suggested that RAGE interactions with ligands such as the S100/calgranulins play a proximal role in the inflammatory cascade. For example, prevention of NF- $\mathrm{KB}$ activation and the generation of IL- 2 and TNF- $\alpha$ are critical early events leading to delayed-type hypersensitivity (9). Prompted by our observations on delayed-type hypersensitivity, we extended our studies to other models, such as colitis in IL-10-null mice, collagen-induced arthritis, and experimental autoimmune encephalitis. Administration of sRAGE to IL-10-deficient mice suppresses the development of colitis and associated findings. Indeed, histologic studies show decreased cryptitis, diminished nuclear binding activity for NF- $\mathrm{KB}$ in nuclear extracts of colons, and reduced systemic levels of TNF- $\alpha(9)$. Our pilot studies in collagen-induced arthritis demonstrate a similar suppression of the inflammatory response in animals treated with sRAGE. In each case, it appears that antagonism of RAGE interferes with the effector stage of the disease, rather than the initial priming of lymphocytes. Further studies will be required to elucidate how RAGE contributes to the biology of lymphocytes and mononuclear phagocytes, but the current findings suggest that RAGE-S100/calgranulin interaction represents a potentially important axis capable of amplifying immune/inflammatory responses.

\section{RAGE and amphoterin: implications \\ for tumor biology}

Amphoterin is a high-mobility group I nonhistone chromosomal DNA-binding protein (38). As such, it is found in the nucleus, although its role in chromosomal structure and function is not fully understood. As with the S100/calgranulins, amphoterin is found, unexpectedly, in the extracellular space, where it is prominent during certain stages of development (38). ECM-associated amphoterin promotes neurite outgrowth and provides a surface for assembly of protease complexes in the fibrinolytic system, which can contribute to cell mobility. We have found that amphoterin-induced neurite outgrowth is mediated by RAGE (36), and studies of Huttunen et al. (38) have revealed that ligation of this receptor activates two small GTPases of the Rho family, Cdc42 and Rac, which are implicated in cytoskeletal remodeling in cell movement.

In view of increased expression of amphoterin and RAGE in tumors, we considered that the tumor bed might be an ideal locus to study the effect of amphoterin-RAGE interaction on cell migration and invasion (10). In a primary tumor model (C6 glioma), administration of anti-RAGE $\mathrm{F}\left(\mathrm{ab}^{\prime}\right)_{2}$ or sRAGE partially inhibits local tumor growth. Furthermore, stable transfectants of C6 glioma cells that overexpress wild-type RAGE produced larger and more invasive tumors than do parental C6 cells. Conversely, cells expressing a dominant negative form of RAGE or sRAGE produce only very small tumors, indicating that blockade of RAGE function strongly suppresses tumor growth, as has also been observed in the Lewis lung metastasis model (10). Moreover, in that latter model, when animals are treated with sRAGE at the time the primary tumor is excised, lung metastases are barely detectable, although they are readily apparent in vehicle-treated mice. Finally, in an endogenous tumor model, spontaneously arising papillomas in mice expressing v-Ha-ras (39), administration of sRAGE markedly suppressed the development of papillomas. The major effect of RAGE appears to be on tumor cell migration and invasiveness, rather than apoptosis or proliferation, although RAGE blockade can also reduce cell growth. Consistent with this impression, increased levels of MMPs 2 and 9, which are closely associated with tumor cell invasion, are detected in tumors derived from cells bearing wild-type RAGE but not in those in which RAGE is blocked.

In vitro studies were performed to gain further insights into mechanisms underlying the antitumor effect of blocking RAGE (10). Cultured C6 glioma cells show a striking difference in morphology and activation of intracellular signalling pathways when plated on amphoterin-coated matrices, depending on whether they overexpress wild-type RAGE or carry dominant negative RAGE. The former cells adhere to the surface and extend long processes, whereas the latter are rounded with little extension of cellular processes. In cellular invasion assays, C6 cells bearing wild-type RAGE show enhanced penetration of Matrigel. In the presence of amphoterin they activate the mitogen-activated protein kinase pathway components $\mathrm{p} 38$, stress-activated protein kinase, and extracellular signal-regulated protein kinase (erk1/2), a response that is strikingly diminished in cells bearing DN-RAGE or expressing sRAGE. These studies suggest that RAGE binding to amphoterin in the tumor bed enhances cell migration and invasion. In contrast, RAGE-ligand interactions do not appear to markedly alter tumor cell viability or angiogenesis. RAGE blockade may therefore dovetail well with cytotoxic or antiangiogenic therapies.

\section{Summation and hypotheses}

At first glance, it seems difficult to fathom how one receptor could be involved in an adverse manner, at least with respect to restitution of homeostasis, in so many and such diverse situations. It seems likely that RAGE has escaped evolutionary pressure, or it would have long ago been eliminated or markedly changed. Probably because the RAGE pathway contributes to chronic disorders occurring later in life, the receptor has remained in the genome. For example, the deposition of AGEs in diabetic tissues, the accumulation of amyloid in Alzheimer brain, and the persistent presence of S100/calgranulins at sites of chronic inflammation drive receptor expression and, thereby, the subsequent cellular responses. Here, the ligand appears to dictate the biology of the receptor in that the presence of ligand triggers expression of the receptor and the ensuing cellular response. As far as we know, this response can only be suppressed by intercepting RAGE-ligand interaction, as occurs in the presence of sRAGE or blocking anti- 
bodies to the receptor. Alternatively, RAGE may have been retained in the genome because of as-yet unrecognized cytoprotective properties (see below).

It is important to note that ligand-RAGE interaction can have strikingly different consequences on different cell types. Thus, the interaction of $A \beta$ with RAGE on microglia stimulates chemotaxis and expression of cytokines (for example, TNF- $\alpha$ ) (27). On neurons and neuron-like RAGE-bearing cells, shortly after exposure to ligands, NF- $\kappa B$ is activated and leads to heightened expression of cell stress-associated proteins. At later times, the same cells may be forced down an apoptosis-like pathway (33). This difference in the outcome may reflect the capacity of each cell type to respond to cell stress signals - for example, those generated by oxidants and/or other toxic intermediates - or may arise from cell type-specific expression of coreceptors or signaling molecules.

Our findings suggest that RAGE will be primarily involved not as a causative or initiating factor for diseases such as diabetes or Alzheimer disease but rather as a mediator of the subsequent effects of chronic cellular activation such as cellular dysfunction and tissue destruction. Thus, antagonism of RAGE might be advantageous for arresting or ameliorating a range of pathophysiologically relevant disorders. In this regard, it will be important to understand the fine structure of receptor-ligand interaction. For example, although the macromolecular ligands of RAGE cross-compete for binding to the receptor, it is unclear whether they interact with identical portions of the V-domain, or whether there is a large binding pocket with multiple closely situated subdomains optimally fitting different ligand-bearing structures.

It is to be expected that there will be commonalities of structure that these otherwise seemingly diverse ligands assume in order to interact with RAGE, and indeed, all of the ligands studied so far appear to be multivalent. For instance, nonenzymatic glycation can modify more than one group on a single macromolecule. The formation of intermolecular crosslinks by the same process (4) further increases the number of potential modification sites on a particular protein. Similarly, S100/calgranulins readily aggregate, and their activity has most often been observed after formation of a multimeric structure (37). Finally, $\beta$-sheet fibrils are ideally suited to rearrange the surface topology of cells with which they interact because of the density of possible ligand sites on any individual fiber. In each case, it is important to consider the possibility that one of these ligand-bearing structures crosslinks RAGE on one cell type, maximizing the cellular response, or that it might bridge two RAGE-bearing cells. In the latter case, RAGE might function as a costimulatory molecule, perhaps by bridging mononuclear phagocytes and $\mathrm{T}$ lymphocytes via S100/calgranulins that engage RAGE proteins on the surface of each cell. Such an interaction would be analogous to B7-1/B7-2 interaction with CD28 (in the case of macrophage lymphocyte interaction in immunity) (40), except that the relative abundance and soluble nature of S100/calgranulins might provide a certain level of nonspecificity (and augmen- tation) of cellular activation in the ensuing immune/inflammatory response. Another possible consequence of this promiscuous multivalency of RAGE ligands might be the recruitment of coreceptors, which could contribute to subsequent cellular events. For example, $A \beta$ interacts with several cell surface binding sites in addition to RAGE (27); the latter include type A scavenger receptors (29), $\alpha-7$ nicotinic acetylcholine receptors (30), heparin-like proteoglycans (32), and neurotrophin receptors (31), among others. Future studies are in progress on many levels to address questions regarding structure-function relationships central to the properties of RAGE, and the extent to which the receptor regulates cell-cell crosstalk, as well as possible interactions with coreceptors.

It is also to be expected that there are situations where the receptor will exert a beneficial effect, probably via the same inflammatory pathways. For example, it is very possible that an ancient structure borne by microorganisms, but not yet identified, will interact with RAGE, consistent with the placement of this receptor in the innate immune system. RAGE probably does not function as an endocytic pattern-recognition receptor like SR-A (see Platt and Gordon, this Perspective series, ref. 41; and refs. 1, 2, 42, 43) but rather may serve as a signaling receptor that can drive subsequent immune/inflammatory events, as the Toll-like receptors, another family of pattern-type receptors, are believed to do (43). RAGE may also exert a beneficial role in limited inflammatory responses, in which ligands are present only briefly. In peripheral nerve injury, such as a sciatic nerve crush model which we have begun to study, blocking ligand-RAGE interaction actually delays tissue repair (44). In this context, nerve injury is associated with a rapid and transient rise in $\mathrm{S} 100 /$ calgranulins and amphoterin at the site of damage, resulting in local upregulation of RAGE. The enhanced expression of RAGE in the injured nerve follows closely the time course for ligands of the receptor and, thus, is also quite brief. Blocking RAGE-ligand interaction in the injured nerve appears to damp critical inflammatory mechanisms that are necessary for disposal of myelin debris at the damage site and subsequent remyelination, as well as for decreasing neurite outgrowth. Although this model of peripheral nerve injury may prove limited in its applicability to human disease, it does suggest a way in which RAGE-mediated processes can be beneficial in repair. Though there is much more to be learned about the contribution of RAGE to health and disease, these results provide an outline mapping properties of an intriguing multiligand member of the immunoglobulin superfamily of cell surface molecules.

\section{Acknowledgments}

This work was supported by grants from the USPHS (HL-60901, AG-17490, AG-16223), the Juvenile Diabetes Research Foundation, the Greenwall Foundation, the Burroughs Wellcome Trust, and the Surgical Research Fund. Please note that, due to restrictions on the number of references permitted by $J C I$ policy, the reference list is quite narrow; it is not meant in any way to diminish the contribution of references not cited. 
1. Hampton, R., Golenbock, D., Penman, M., Krieger, M., and Raetz, C. 1991. Recognition and plasma clearance of endotoxin by scavenger receptors. Nature. 352:342-344.

2. Fraser, I., Koziel, H., and Ezekowitz, R. 1998. The serum mannose-binding protein and the macrophage mannose receptor are pattern recognition molecules that link innate and adaptive immunity. Semin. Immunol. 10:363-372.

3. Medzhitov, R., and Janeway, C. 2000. Innate Immunity. N. Engl. J. Med. 343:338-344.

4. Brownlee, M. 1995. Advanced glycosylation in diabetes and aging. Annu. Rev. Med. 46:223-234.

5. Miyata, T., et al. 1996. RAGE mediates the interaction of AGE-beta-2microglobulin with human mononuclear phagocytes via an oxidantsensitive pathway: implications for the pathogenesis of dialysis-related amyloidosis. J. Clin. Invest. 98:1088-1094.

6. Schmidt, A.-M., Yan, S.-D., and Stern, D.M. 1995. The dark side of glucose. Nat. Med. 1:1002-1004.

7. Schmidt, A.M., et al. 1992. Isolation and characterization of binding proteins for advanced glycosylation endproducts from lung tissue which are present on the endothelial cell surface. J. Biol. Chem. 267:14987-14997.

8. Neeper, M., et al. 1992. Cloning and expression of RAGE: a cell surface receptor for advanced glycosylation end products of proteins. J. Biol. Chem. 267:14998-15004.

9. Hofmann, M., et al. 1999. RAGE mediates a novel proinflammatory axis: the cell surface receptor for S100/calgranulin polypeptides. Cell. 97:889-901.

10. Taguchi, A., et al. 2000. Blockade of RAGE/amphoterin suppresses tumor growth and metastases. Nature. 405:354-360.

11. Li, J., and Schmidt, A.-M. 1997. Characterization and functional analysis of the promoter of RAGE. J. Biol. Chem. 272:16498-16506.

12. Li, J., Qu, W., and Schmidt, A.-M. 1998. Sp1 binding elements in the promoter of RAGE are essential for amphoterin-mediated gene expression in cultured neuroblastoma cells. J. Biol. Chem. 273:30870-30878.

13. Tanaka, N., et al. 2000. RAGE is induced by the glycation products themselves and TNF-alpha through NF-kB and by $17 \beta$-estradiol through Sp1 in human vascular endothelial cells. J. Biol. Chem. 275:25781-25790.

14. Kislinger, T., et al. 1999. N(epsilon)-(carboxymethyl)lysine adducts of proteins are ligands for RAGE that activate cell signaling pathways and modulate gene expression. J. Biol. Chem. 274:31740-31749.

15. Bierhaus, A., et al. 1997. AGE-mediated induction of tissue factor in cultured endothelial cells is dependent on RAGE. Circulation. 46:1481-1490

16. Yamagishi, S., et al. 1997. AGE-driven angiogenesis in vitro. Induction of the growth and tube formation of human microvascular endothelial cells through autocrine vascular endothelial growth factor. J. Biol. Chem. 272:8723-8730.

17. Yamagishi, S., Fujimori, H., Yonekura, H., Yamamoto, Y., and Yamamoto, H. 1998. AGEs inhibit prostacyclin production and induce PAI-1 in human microvascular endothelial cells. Diabetologia. 41:1435-1441.

18. Wautier, J.L., et al. 1996. Receptor-mediated endothelial cell dysfunction in diabetic vasculopathy: soluble RAGE blocks hyperpermeability. J. Clin. Invest. 97:238-243.

19. Schmidt, A.-M., et al. 1994. RAGE has a central role in vessel wall interactions and gene activation in response to AGEs. Proc. Natl. Acad. Sci. USA. 91:8807-8811.

20. Schmidt, A.-M., et al. 1995. Advanced glycation endproducts interacting with their endothelial receptor induce expression of VCAM-1 in cultured human endothelial cells and in mice. J. Clin. Invest. 96:1395-1403.

21. Yan, S.D., et al. 1994. Enhanced cellular oxidant stress by the interaction of AGEs with their receptors/binding proteins. J. Biol. Chem. 269:9889-9897.

22. Schmidt, A.M., et al. 1993. Regulation of human mononuclear phago- cyte migration by cell surface binding proteins for AGEs. J. Clin. Invest 91:2155-2168

23. Park, L., et al. 1998. Suppression of accelerated diabetic atherosclerosis by soluble receptor for AGE (sRAGE). Nat. Med. 4:1025-1031.

24. Wendt, T., et al. 2000. Accelerated atherosclerosis and vascular inflammation develop in apoE null mice with type 2 diabetes. Circulation. 102(Suppl. II):1124. (Abstr.)

25. Goova, M., et al. 2001. Blockade of RAGE restores effective wound healing in diabetic mice. Am. J. Pathol. 159:513-525.

26. Lalla, E., et al. 2000. Blockade of RAGE suppresses periodontitis-associated alveolar bone loss in diabetic mice. J. Clin. Invest. 105:1117-1124.

27. Yan, S.-D., et al. 1996. RAGE and amyloid-beta peptide neurotoxicity in Alzheimer's disease. Nature. 382:685-691.

28. Yan, S.-D., et al. 2000. Receptor-dependent cell stress and amyloid accumulation in systemic amyloidosis. Nat. Med. 6:643-651.

29. Paresce, D.M., Ghosh, R.N., and Maxfield, F.R. 1996. Microglial cells internalize aggregates of the Alzheimer's disease amyloid beta-protein via a scavenger receptor. Neuron. 17:553-565.

30. Wang, H.-Y., et al. 2000. A $\beta(1-42)$ binds to $\alpha-7$ nicotinic acetylcholine receptor with high affinity. J. Biol. Chem. 275:5626-5632.

31. Yaar, M., et al. 1997. Binding of beta-amyloid to the $\mathrm{p} 75$ neurotrophin receptor induces apoptosis. J. Clin. Invest. 100:2333-2340.

32. Giulian, D., et al. 1998. The HHQK domain of beta-amyloid provides a structural basis for the immunopathology of Alzheimer's disease. J. Biol. Chem. 273:29719-29726.

33. Sousa, M., et al. 2001. Familial amyloid polyneuropathy: RAGE-dependent triggering of neuronal inflammatory and apoptotic pathways. $J$. Neurosci. In press.

34. Stern, D., et al. 2000. Enhanced neuronal stress in double transgenic mice with targeted overexpression of RAGE and mutant APP. Society for Neuroscience Abstract 351-\#491.14.

35. Kumar, S., et al. 2000. RAGE at the blood-brain barrier mediates neurovascular dysfunction caused by amyloid $\beta 1-40$ peptide. Society for Neuroscience Abstract 141-\#275.19.

36. Hori, O., et al. 1995. RAGE is a cellular binding site for amphoterin: mediation of neurite outgrowth and co-expression of RAGE and amphoterin in the developing nervous system. J. Biol. Chem. 270:25752-25761.

37. Schafer, B., and Heizmann, C. 1996. The S100 family of EF-hand calcium-binding proteins: functions and pathology. Trends Biochem. Sci. 21:134-140.

38. Huttunen, H., Fages, C., and Rauvala, H. 1999. RAGE-mediated neurite outgrowth and activation of NF-kB require the cytoplasmic domain of the receptor but different downstream signaling pathways. J. Biol. Chem. 274:19919-19924.

39. Leder, A., Kuo, A., Cardiff, R., Sinn, F., and Leder, P. 1990. V-Ha-ras transgene abrogates the initiation step in mouse skin tumorigenesis: effects of phorbol esters and retinoic acid. Proc. Natl. Acad. Sci. USA. 87:9178-9182.

40. June, C., Bluestone, J., Nadler, L., and Thompson, C. 1994. B7 and CD28 receptor families. Immunol. Today. 15:321-331.

41. Platt, N., and Gordon, S. 2001. Is the class A macrophage scavenger receptor (SR-A) multifunctional? - The mouse's tale. J. Clin. Invest. 108:649-654.

42. Krieger, M., and Herz, J. 1994. Structures and functions of multiligand lipoprotein receptors: macrophage scavenger receptors and LDL receptor-related protein. Annu. Rev. Biochem. 63:601-637.

43. Rock, F., Hardiman, G., Timans, J., Kastelein, R., and Bazan, J. 1998. A family of human receptors structurally related to Drosophila Toll. Proc. Natl. Acad. Sci. USA. 95:588-593.

44. Rong, L., et al. 2000. RAGE and its ligands, EN-RAGEs and amphoterin are expressed in injured peripheral nerve and modulate regeneration in a murine model of unilateral sciatic nerve crush. Society for Neuroscience Abstract 58-\#114.4 